\title{
7. Evaluación reumatológica del paciente con enfermedad pulmonar intersticial
}

\author{
VERÓNICA WOLFF C.* y OMAR VALENZUELA L.**
}

\section{Rheumatological evaluation of the patient with interstitial lung disease}

Connective Tissue Diseases (CTD) comprise a heterogeneous group of multisystemic pathologies of autoimmune origin. Interstitial lung disease (ILD) associated with CTD (CTD-ILD) is common and and it worsens the prognosis of CTD. CTD-ILD represent approximately $15-30 \%$ of the universe of ILD and have the same histopathological and radiological forms described for idiopathic ILD. This highlights the importance of routinely incorporate a rheumatologist into the multidisciplinary committee for the diagnosis and management of ILD.

Key words: Lung Diseases; Interstitial; Connective Tissue Diseases; Rheumatologists.

\section{Resumen}

Las Enfermedades del Tejido Conectivo (ETC) comprenden un grupo heterogéneo de patologías multisistémicas de origen autoinmune. La Enfermedad pulmonar intersticial (EPI) asociada a ETC (EPI-ETC) es frecuente y empeora el pronóstico de la ETC. Las EPI-ETC representan aproximadamente 15-30\% del total las EPI y se presentan con las mismas formas histopatológicas y radiológicas descritas para las EPI idiopáticas. Esto pone en evidencia la importancia de incorporar en forma rutinaria a reumatología en el comité multidisciplinario para el diagnóstico y manejo de las EPI.

Palabras clave: Enfermedades pulmonares intersticiales; Enfermedades del tejido conectivo; Reumatólogos.

Las Enfermedades del Tejido Conectivo (ETC) comprenden un grupo heterogéneo de patologías sistémicas de origen autoinmune (Tabla 1) que con frecuencia comprometen el aparato respiratorio. Una de las principales formas de compromiso pulmonar es la Enfermedad Pulmonar Intersticial (EPI). Ésta se presenta con las mismas formas histopatológicas descritas para las EPI idiopáti$\operatorname{cas}^{1,2}$.

Las ETC que con mayor frecuencia presentan compromiso pulmonar intersticial son esclerosis sistémica: SCL (50\%-60\%), Artritis Reumatoidea: AR (10\%-30\%) y el espectro de las Miopatías Inflamatorias Idiopáticas: MII (20\%-86\%). En este último grupo el compromiso pulmonar está claramente determinado por el perfil de autoanticuerpos del paciente ${ }^{2}$.
Entre $15 \%$ y $30 \%$ de los pacientes que se presentan con EPI de causa desconocida tienen una ETC no diagnosticada ${ }^{3}$. La respuesta al tratamiento inmunosupresor y el pronóstico de las EPI secundarias a ETC es globalmente mejor que en las formas idiopáticas de $\mathrm{EPI}^{4}$, principalmente, comparado con la fibrosis pulmonar idiopática (FPI), patología en la cual se ha demostrado la inutilidad y potencial daño de la terapia inmunosupresora $^{5}$.

Si bien en el contexto de ETC-EPI se presenta la mayoría de las variantes histopatológicas descritas para las EPI idiopáticas, la más frecuente en ETC es la Neumonia Intersticial no específica: NINE $^{6,7}$. Excepciones a esta situación encontramos en AR, en la cual la variante NIU es la más frecuente, y en las MII la sobreposición de

* Hospital del Salvador. Instituto Nacional del Tórax. Profesor Asistente de la Universidad de Chile. Clínica Las Condes. Santiago, Chile.

** Hospital Padre Hurtado. Clínica Alemana de Santiago. Universidad del Desarrollo. Santiago, Chile. 
Tabla 1. Principales Enfermedades de Tejido Conectivo con compromiso pulmonar intersticial

\begin{tabular}{l}
\hline Lupus Eritematoso Sistémico (LES) \\
Esclerosis Sistémica o Esclerodermia (SCL) \\
\hline Artritis Reumatoide (AR) \\
Síndrome de Sjögren Primario (SS) \\
Miopatías Inflamatorias idiopáticas (MII): Polimiositis, \\
Dermatomiositis, Síndrome Antisintetasas \\
\hline Enfermedad Mixta del Tejido Conectivo (EMTC) \\
$\begin{array}{l}\text { Enfermedad Indiferenciada del Tejido Conectivo } \\
\text { (EITC) }\end{array}$ \\
Vasculitis sistémicas asociadas a Anticuerpos anti cito- \\
plasma de neutrófilos (ANCA)
\end{tabular}

NINE/NO (neumonia en organización) es muy característica $^{8,9}$

Por lo anterior, es fundamental la integración rutinaria del reumatólogo dentro del equipo multidisciplinario, quien podrá ayudar a descartar alguna de estas condiciones antes de diagnosticar una EPI idiopática.

\section{Contextos asociados a ETC-EPI}

La EPI en un paciente con ETC se da, principalmente, en dos escenarios:

1.1. Paciente con ETC conocida preexistente que desarrolla EPI en el transcurso de su enfermedad reumatológica.

1.2. Paciente que debuta con EPI sin diagnóstico previo de ETC.

En el primer escenario, el papel del reumatólogo será trabajar junto al neumólogo en determinar si la EPI es parte de las manifestaciones sistémicas de la ETC u obedece a otras causas (infecciosas, ambientales, etc.). Además, debe participar en el diseño conjunto de una terapia que incorpore adecuadamente el tratamiento de la EPI y las manifestaciones extrapulmonares de la ETC.

El segundo escenario representa un desafío clínico importante en el contexto ETC-EPI, ya que en algunas ocasiones las manifestaciones extrapulmonares serán muy sutiles y puede que el paciente no cumpla con los criterios de clasificación para una ETC en particular a pesar de tener claros elementos de autoinmunidad en el cuadro clínico. Tradicionalmente en reumatología, situaciones como esta se han clasificado como Enfermedades Indiferenciadas del Tejido Conectivo, y en el
Tabla 2. Categorías de Enfermedades Pulmonares Intersticiales que requieren evaluación reumatológica

\section{Mujeres menores de 50 años}

2. Pacientes con manifestaciones extratorácicas altamente sugerentes de enfermedad del tejido conectivo:

- Fenómeno de Raynaud

- Artritis de manos y muñecas

- Telangectasias periungueales, mucosas, faciales o palmares

- Úlceras digitales

- Esclerodactilia, manos edematosas ("puffy")

- Manos de mecánico

- Parotidomegalia, queratoconjuntivitis, Sicca demostrada

- Hipomotilidad esofágica demostrada

3. Todos los casos de NINE, NO, NIL, NIA o compromiso multicompartamental

4. Serología sugerente de ETC

- ANA o FR (+) a título alto

- ANA con patrón nucleolar en cualquier título

- Otros autoanticuerpos muy específicos (+): anti CCP, anti SCL-70, anti Ro, anti La, anti Sm, anti DNA, anti RNP, antisintetasas, anti MDA-5

Adaptado y modificado de Fischer A et al. ${ }^{11}$. NINE: neumonía intersticial no específica, NO: neumonía en organización, NIL: neumonía intersticial linfoide, NIA: neumonía intersticial aguda (NIA), ANA: anticuerpo antinuclear; FR: factor reumatoideo, CCP: péptido cíclico citrulinado.

caso particular de ETC-EPI, existe un consenso reciente que permite clasificar estos casos como "Neumonía intersticial con elementos de autoinminidad" o "IPAF" por sus siglas en inglés ${ }^{10}$, lo que debe ser interpretado como un diagnóstico de trabajo más que un diagnóstico en sí mismo.

No existe un algoritmo universalmente aceptado para realizar el diagnóstico de ETC en el contexto de EPI. No siempre es posible contar con una evaluación reumatológica de todos los pacientes, pero hay algunas categorías de EPI en que ésta es necesaria (Tabla 2). A continuación, se explicitan elementos que sugieren ETC y la intervención de un reumatólogo:

\section{Subtipo de Enfermedades Pulmonares Intersticiales}

Cualquier compromiso No-NIU asociado a compromiso multicompartamental torácico genera una alta sospecha de ETC subyacente no diagnosticada previamente.

\section{Elementos de la historia clínica y del examen} fisico altamente sugerentes de ETC

a) Pacientes de sexo femenino y más jóvenes $(<50$ años $)$. 
b) Síntomas altamente sugerentes de autoinmunidad. Entre estos, el más importante es el fenómeno de Raynaud, muy prevalente en el espectro de SCL/MII. Cuando exista disponibilidad puede ser útil la capilaroscopía del lecho ungueal, examen no invasivo y relativamente sencillo de realizar, que permite detectar patrones patológicos de los capilares del lecho ungueal que están claramente relacionados a algunas ETC, principalmente, SCL, Enfermedad mixta del tejido conectivo: EMTC y espectro MII.

c) Lesiones isquémicas ulceradas de los pulpejos o cicatrices ("pitting scars").

d) Edema de los dedos de las manos (manos "puffy") o endurecimiento de la piel de los dedos (esclerodactilia) con o sin compromiso de brazos, cara y tronco, elevan la sospecha de una ETC en el espectro de SCL.

e) Presencia de telangectasias periungueales, en palmas de las manos, cara y mucosa oral, sugerentes de SCL.

f) Artritis que compromete especialmente las manos y muñecas o la historia de dolor articular de manos y muñecas reciente, con rigidez matinal de más de $1 \mathrm{~h}$.

g) Debilidad muscular proximal de cintura escapular y pélvica deben hacer sospechar una ETC en el espectro de las MII. Sin embargo, no es infrecuente un mínimo o ausente compromiso del músculo (formas "amiopáticas"). En esta circunstancia, es relevante la presencia de autoanticuerpos específicos o asociados a MII.

h) Presencia de "manos de mecánico", rash característico del llamado "Síndrome Antisintetasa". Este corresponde a un subgrupo entre las MII, que se caracteriza por un conjunto de síntomas con frecuencia variable: fiebre, artritis de manos, rash tipo "manos de mecánico", fenómeno de Raynaud, miositis $(<50 \%)$, muy alta prevalencia de EPI ( $>70-90 \%)$ y presencia de anticuerpos antisintetasas.

i) Pápulas de Gottron, rash heliotropo y eritema en esclavina (del escote) orientan a una dermatomiositis.

j) La inflamación parotídea recurrente, asociada a síntomas de sicca con o sin queratoconjuntivitis orientan a un Síndrome de Sjögren.

\section{Laboratorio y Serología inmunológica}

Como parte del descarte de una ETC y eventual compromiso de otros órganos, todos los pacientes deben someterse a exámenes de laboratorio básicos que incluyan: hemograma con VHS, función renal, hepática y orina completa. En pacientes en los que exista la sospecha clínica

Tabla 3. Principales autoanticuerpos y Enfermedades del Tejido Conectivo asociadas

\begin{tabular}{|ll|}
\hline Anticuerpo & ETC asociada \\
\hline ANA principalmente $>1 / 320$ & Muchas: SCL, EMTC, LES, SS, AR, etc \\
\hline FR título alto $>2$ veces el basal & AR, SS, LES \\
\hline Anti CCP & AR \\
ANA patrón anticentrómero & SCL limitada \\
\hline ANA patrón nucleolar & SCL \\
Anti Ro (SSA) & SS, LES, Sd. antisintetasas \\
\hline Anti La (SSB) & SS, LES \\
Anti Sm & LES \\
\hline Anti RNP & EMTC, LES \\
Anti DNA nativo & LES \\
Anti topoisimerasa (Scl-70) & SCL \\
Anti sintetasas (Jo-1; PL-7; PL-12; OJ; EJ) & Sd. antisintetasas \\
\hline Anti PM/Scl 75 y 100, anti Ku & Sd. sobreposición SCL/MII (fenotipo similar a Sd. antisintetasas) \\
\hline Anti Th/to & SCL \\
\hline Anti U3RNP & SCL \\
\hline Anti MDA-5 & Dermatomiositis amiopática (fenotipo similar a Sd. antisintetasas) \\
\hline ANCA & Vasculitis ANCA asociada \\
\hline
\end{tabular}

Adaptado y modificado de Fischer A, et $\mathrm{al}^{11}$. SLC: esclerodermia; EMTC: enfermedad pulmonar mixta del tejido conectivo; LES: lupus eritematoso sistémico; SS: Síndrome de Sjögren; AR: artritis reumatoide; MII: miopatías inflamatorias idiopática. 
Tabla 4. Autoanticuerpos sugeridos de acuerdo al patrón de Enfermedad Pulmonar Intersticial

\begin{tabular}{|ll|}
\hline Patrón de EPI & Anticuerpo sugerido \\
\hline NIU & FR, CCP, ANA, perfil ENA (Ro, La, Sm, RNP, ScL70, Jo-1), ANCA \\
NINE, NO, NINE/NO & ANA, DNA, perfil ENA (Ro, La, Sm, RNP, Scl70, Jo-1), panel de miositis, MDA-5 \\
\hline NIL & ANA, FR, perfil ENA, DNA, CCP \\
NIA/DAD & ANA, perfil ENA, DNA, panel de miositis, MDA-5 \\
Enf bronquiolar & ANA, FR, perfil ENA, CCP \\
\hline
\end{tabular}

Adaptado y modificado de Doyle T, et al. ${ }^{2}$. NIU: neumonía intersticial usual; NINE: neumonía intersticial no específica; NO: neumonía en organización; NIL: neumonía intersticial linfoide; NIA: neumonía interticial aguda; DAD: daño alveolar difuso.

de ETC en el espectro MII, se debe añadir la medición de creatinfosfoquinasa (CK).

La serología inmunológica es un elemento importante de apoyo en el diagnóstico de ETC o IPAF, especialmente útil cuando la ETC no es evidente. En la Tabla 3 se mencionan los autoanticuerpos más relevantes asociados a las distintas ETC. La elección del panel de autoanticuerpos a solicitar dependerá de la sospecha clínica en cada caso. En la Tabla 4 se sugiere cuáles autoanticuerpos pedir, de acuerdo con el tipo de EPI que presenta el paciente.

El ANA debe ser idealmente realizado por la técnica de inmunofluorescencia indirecta (IFI), no por ELISA, ya que la IFI permite la descripción del patrón de inmunofluorescencia del ANA, asociado a diversas ETC. Por ejemplo, el patrón "nucleolar" se asocia específicamente al espectro de SCL y el patrón "anticentrómero" a la SCL variedad limitada.

\section{Hallazgos anátomo-patológicos}

Entre los hallazgos patológicos altamente sugerentes de ETC destacan los siguientes: patrón de neumonía intersticial no específica (NINE), neumonía en organización (NO), neumonía intersticial linfoide (NIL), neumonía intersticial aguda (NIA)/ daño alveolar difusa (DAD), acúmulos linfoides con centros germinales, infiltrado linfoplasmocitario prominente, folículos linfoides, compromiso de otros compartimentos en forma simultánea (pleura, pericardio, enfermedad bronquiolar, bronquiectasias, vasculopatía pulmonar, etc).

\section{Bibliografía}

1.- LAMBKIN C, BERGIN C, SEALERS T, WALLER B. Interstial lung diseases in collagen vascular diseases. Eur Resp J 2001; 32 (suppl): S69-80.

2.- DOYLE TJ, DELLARIPA PF. Lung manifestations in the rheumatic diseases. Chest 2017; 152: 1283-95.

3.- MITOO S, GELBER AC, CHRISTOPHER-STINE
L, HORTON MR, LECHTZIN N, DANOFF SK. Ascertainment of collagen vascular disease in patients presenting with interstitial lung disease. Respir Med 2009; 103: 1152-8.

4.- PARK JH, KIM DS, PARK IN, JANG SJ, KITAICHI M, NICHOLSON AG, et al. Prognosis of fibrotic interstitial pneumonia: idiopathic versus collagen vascular disease-related subtypes. Am J Respir Care Med 2007; 175: 705-11.

5.- RAGHU G, ANSTROM KJ, KING TE JR, LASKY JA, MARTÍNEZ FJ, Idiopathic Pulmonary Fibrosis Clinical Research Network. Prednisone, azathioprine and $\mathrm{N}$-acetylcysteine for pulmonary fibrosis. $\mathrm{N}$ Engl $\mathrm{J}$ Med 2012; 366: 1968-77.

6.- TANSEY D, WELLS AU, COLBY TV, IP S, NIKOLAKOUPOLOU A, DU BOIS RM, et al. Variations in histological patterns of interstitial pneumonia between connective tissue disorders and their relationship to prognosis. Histopathology 2004; 44: 585-96.

7.- VIVERO M, PRADERA RF. Histopathology of lung disease in the connective tissue diseases. Rheum Dis Clin North Am 2015; 41: 197-211.

8.- LAKE F, PROUDMAN S. Rheumatoid arthritis and lung disease: from mechanisms to a practical approach. Semin Respir Crit Care Med 2014; 35: 222-38.

9.- CAVAGNA L, TRALLERO-ARAGUÁS E, MELONI F, CAVAZZANA I, ROJAS-SERRANO J, et al. Influence of Antisynthetase Antiboides Syndrome Clinical Spectrum Time Course. J Clin Med 2019; 8: 2013.

10.- FISCHER A, ANTONIOU KM, BROWN KK, CADRANEL J, CORTE TJ, DU BOIS RM, et al. An official European Respiratory Society/American Thoracic Society research statement: interstitial pneumonia with autoimmune features. Eur Respir J 2015; 46: 976-87.

11.- FISCHER A, LEE J, COTTIN V. ILD evaluation: Detecting connective tissue disease. Respiration 2015; 90: 177-84.

Correspondencia a:

Dra. Verónica Wolff C.

Email: vero.wolff.c@gmail.com 